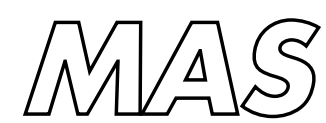

Modelling, Analysis and Simulation 
CWI is the National Research Institute for Mathematics and Computer Science. It is sponsored by the Netherlands Organization for Scientific Research (NWO).

$\mathrm{CWI}$ is a founding member of ERCIM, the European Research Consortium for Informatics and Mathematics.

CWI's research has a theme-oriented structure and is grouped into four clusters. Listed below are the names of the clusters and in parentheses their acronyms.

Probability, Networks and Algorithms (PNA)

Software Engineering (SEN)

Modelling, Analysis and Simulation (MAS)

Information Systems (INS)

Copyright @ 2001, Stichting Centrum voor Wiskunde en Informatica

P.O. Box 94079, 1090 GB Amsterdam (NL)

Kruislaan 413, 1098 SJ Amsterdam (NL)

Telephone +31205929333

Telefax +31205924199

ISSN 1386-3703 


\title{
Control of Spatially Heterogeneous and Time-Varying Cellular Reaction Networks: A New Summation Law
}

\author{
Mark A. Peletier \\ Centrum voor Wiskunde en Informatica, Amsterdam, The Netherlands \\ Hans V. Westerhoff \\ Vrije Universiteit, Amsterdam, The Netherlands \\ Boris N. Kholodenko \\ Thomas Jefferson University, Philadelphia, USA
}

\begin{abstract}
A hallmark of a plethora of intracellular signaling pathways is the spatial separation of activation and deactivation processes that potentially results in precipitous gradients of activated proteins. The classical Metabolic Control Analysis (MCA), which quantifies the influence of an individual process on a system variable as the control coefficient, cannot be applied to spatially separated protein networks. The present paper unravels the principles that govern the control over the fluxes and intermediate concentrations in spatially heterogeneous reaction networks. Our main results are two types of the control summation theorems. The first type is a non-trivial generalization of the classical theorems to systems with spatially and temporally varying concentrations. In this generalization, the process of diffusion, which enters as the result of spatial concentration gradients, plays a role similar to other processes such as chemical reactions and membrane transport. The second summation theorem is completely novel. It states that the control by the membrane transport, the diffusion control coefficient multiplied by two, and a newly introduced control coefficient associated with changes in the spatial size of a system (e.g., cell), all add up to one and zero for the control over flux and concentration. Using a simple example of a kinase/phosphatase system in a spherical cell, we speculate that unless active mechanisms of intracellular transport are involved, the threshold cell size is limited by the diffusion control, when it is beginning to exceed the spatial control coefficient significantly.
\end{abstract}

2000 Mathematics Subject Classification: 35B30, 35J60, 35K55

Keywords and Phrases: Metabolic control analysis, signal transduction, diffusive gradients, protein kinase, phosphatase

Note: This work was partially carried out under CWI Research Theme MAS1 'Applied Analysis and Scientific Computing'

\section{INTRODUCTION}

Extracellular information received by plasma membrane receptors is processed, encoded, and transferred to the nucleus through activation and spatial relocation of multiple signalling components. Receptor activation triggers signalling responses associated with the mobilization of a plethora of adapter and target proteins to the plasma membrane (Haugh and Lauffenburger 1997; Kholodenko et al. 2000b). Proteins activated e.g. by phosphorylation at the cell surface travel to stimulate critical regulatory targets at various cellular sites including the nucleus. During and after the travel, these proteins are inactivated, e.g. through dephosphorylation. For instance, a protein phosphorylated by a plasma-membrane associated protein kinase can be dephosphorylated by a phosphatase in the cytosol or nucleus. The transport between cellular locations, where the activation and inactivation occur, is passive (thermal diffusion), driven by spatial gradients in the concentrations. In some cases, not to be discussed here, motor proteins or endocytosis may be involved (Kholodenko 2002). Previous work estimated that these spatial gradients may be large, and that therefore diffusion may contribute to the control of signal transduction (Brown and Kholodenko 1999; Kholodenko et al. 2000a). 
For spatially homogeneous reaction networks, the control over fluxes and intermediate concentrations has been studied both experimentally and theoretically (reviewed in (Fell 1997)). The control is generally quantified as the extent to which any type of molecular process influences a system variable, such as the flux or concentration. The control coefficients are defined as the ratios of the fractional changes in the system variable to that of the biochemical activity which caused the system change. Mathematically, the stability of a system steady state is required and the changes are considered as infinitesimally small, i.e., the coefficients are expressed as the log to log derivatives (Fell 1997). Important principles underlying the control of biochemical reaction networks in well-stirred reactors have been worked out recently. For instance, it can be shown that a large increase in the activity of a single enzyme does not result in a substantial increase in the flux for almost any metabolic network; marked flux increase can be achieved by a concerted modulation of several pathway reactions (Kacser and Acerenza 1993). In metabolic control analysis (MCA), this result is related to the so-called summation theorem, which states that the sum of the enzyme control coefficients adds up to 1 (Kacser and Burns 1973; Heinrich and Rapoport 1973; Westerhoff and van Dam 1987). There is no rate-limiting enzyme, the control is shared between all network processes.

The present paper unravels the principles that govern the control pattern in spatially heterogeneous cellular networks. We demonstrate new properties of control that result from the spatial aspect of diffusion and separation of signalling reactions. The control summation theorems, relevant for these networks, are formulated and proven.

\section{Methods}

2.1 Description of the system

We consider living metabolic systems consisting of $n$ internal chemical species $Y_{1}, \ldots, Y_{n}$, with concentrations $c_{1}, \ldots, c_{n}$. These species interact via $m$ reactions which can be represented as

$$
\sum_{i=1}^{n} N_{i j}^{+} Y_{i} \rightleftarrows \sum_{i=1}^{n} N_{i j}^{-} Y_{i}, \quad j=1, \ldots, m
$$

where $N_{i j}^{ \pm}$are the dimensionless forward and reverse stoichiometric coefficients for the consumption of species $Y_{i}$ in the forward and reverse reactions $j$. We write $N_{i j}=N_{i j}^{-}-N_{i j}^{+}$for the net stoichiometric component. Reaction $j$ has rate $\alpha_{v_{j}} v_{j}\left(c_{1}, \ldots, c_{n}\right)$, so that in the absence of diffusion the time evolution of the concentrations $c_{i}$ should be given by

$$
\frac{\partial}{\partial t} c_{i}=\sum_{j=1}^{m} N_{i j} \alpha_{v_{j}} v_{j}, \quad i=1, \ldots, n .
$$

$c_{i}$ has the dimension of concentration $[\mathrm{M}]$, or mole number per unit volume. The rate of the reaction 2.1, i.e. $v_{j}$, has the dimension of concentration per unit time $[\mathrm{M} / \mathrm{s}]$. It is a function of the kinetic parameters of the enzyme catalyzing that reaction (or if non-enzymatic of the chemical reaction itself), e.g. $K_{M}$, the concentration of the enzyme, and the concentrations of substrates, products and allosteric modifiers of the reaction. The dimensionless parameter $\alpha_{v_{j}}$ is the tool we shall use to modulate the activity of reaction $v_{j}$.

In this paper we will be interested in cases in which concentrations can not be assumed homogeneous in space inside the systems. For thermodynamic reasons, living systems need to be open for at least some chemical compounds. These substances are transported across the system membrane, usually by transport proteins, i.e. effectively there is a sink or source for some of the species on the system boundary, e.g. the (plasma) membrane. The spatial separation between the sink or source and the chemistry that takes place in the bulk phase then creates spatial variations in concentration.

When the system is not homogeneous and not at steady state, the concentrations need to be specified as functions of time and space (i.e. $c_{i}(x, t)$ ), and boundary fluxes are required. The principal difficulty accompanying the spatial inhomogeneity of the concentrations of any component $Y_{i}$, which makes 
the classical MCA inapplicable, is that the number of 'explicit' variables associated with $Y_{i}$ becomes infinitely large. The reactions are assumed to take place in an enclosed three-dimensional space that is represented by a bounded set of $x_{1}, x_{2}, x_{3}$-coordinates, i.e. the volume $V \subset \mathbb{R}^{3}$. For instance, $V$ can correspond to the cytoplasm or the mitochondrial matrix. Inside $V$ the species react with each other, according to Eq. 2.1; in addition they undergo diffusive transport. The concentrations $c_{i}$ of the species are functions of time $\left(0<t<t_{\text {end }}\right)$ and space $(x \in V)$, and their evolution is governed by the balance (reaction-diffusion) equations (Katchalsky and Curran 1965)

$$
\frac{\partial}{\partial t} c_{i}-\alpha_{D_{i}} D_{i} \Delta c_{i}=\sum_{j=1}^{m} N_{i j} \alpha_{v_{j}} v_{j}, \quad x \in V, \quad 0<t<t_{\text {end }} .
$$

The parameter $D_{i}$ is the diffusion coefficient of species $Y_{i}$. It is considered homogeneous. For Cartesian coordinates the Laplacian $\Delta$ is defined as

$$
\Delta=\frac{\partial^{2}}{\partial x_{1}^{2}}+\frac{\partial^{2}}{\partial x_{2}^{2}}+\frac{\partial^{2}}{\partial x_{3}^{2}}
$$

The operator $\Delta$ has the dimension of $1 / \operatorname{length}^{2}\left[\mathrm{~m}^{-2}\right] . \quad D_{i}$ has the dimension of surface per time $\left[\mathrm{m}^{2} / \mathrm{s}\right]$ and is assumed to be the same throughout volume $V$. In order to assess the importance of diffusion of a substance for the behavior of the system, we shall modulate the diffusion processes. To this aim, $D_{i}$ is multiplied by the dimensionless modulation parameter $\alpha_{D_{i}}$, which has the value of 1 in the reference state.

In addition to Eq. 2.2, which holds in the bulk, represented by $V$, we need to specify the behaviour at the boundary $S$ of the set $V$. For a species $Y_{i}$ for which the membrane is a passive barrier we assume a closed boundary condition,

$$
-\alpha_{D_{i}} D_{i} \frac{\partial c_{i}}{\partial \nu}=0 \quad \text { on } S
$$

The vector $\nu$ is the normal vector of unit length pointing out of $V$, and $\partial c_{i} / \partial \nu$ is the derivative of $c_{i}$ in the direction of $\nu$. This makes the quantity $-\alpha_{D_{i}} D_{i} \partial c_{i} / \partial \nu$ the outward diffusive flux of species $Y_{i}$.

If a species is transported across the boundary of $V$, then the boundary condition takes a similar form, but with non-zero right-hand side,

$$
-\alpha_{D_{i}} D_{i} \frac{\partial c_{i}}{\partial \nu}=\alpha_{f_{i}} f_{i} \quad \text { on } S
$$

where $f_{i}$ is the transport rate per unit surface area. $f_{i}$ can be due to active transport or a (bio)chemical reaction at the membrane surface and may be a function of the concentrations $c_{1}, \ldots, c_{n}$. There is at least one non-zero flux $\alpha_{f_{i}} f_{i}$ (one of which we conveniently assume to correspond to $i=1$ ). Like other $\alpha$ 's, $\alpha_{f_{i}}$ is a parameter that we shall use to modulate the transport activity. This modulation is equivalent to changes in the activity or concentration of membrane enzymes or carriers. We shall refer to the 'flux $J$ through the system' as the magnitude of the net export of $Y_{1}$, integrated over the surface of $V$ and normalized by the surface area:

$$
J=\frac{\alpha_{f_{1}}}{|S|} \int_{S} f_{1} d S
$$

We normalize the total flow with respect to the corresponding surface area to obtain a surfaceaveraged flux. The difference in role of a reaction rate in the bulk phase $\left(v_{j}\right)$ and a transport rate $\left(f_{i}\right)$ is underlined by the difference in the dimensionality - the former is given in volume concentration per time unit (e.g., M/s), whereas the latter is expressed in surface concentration (density) per time unit (e.g., M.m/s). We comprise Eq. 2.3 into Eq. 2.4 by allowing $f_{i}$ to be zero. Some reactions that involve cytoplasmic substances only occur in the membrane or at the membrane surface. This is equivalent to the substrate of the reaction being exported and the product of the reaction being imported, and will be treated as such. 
2.2 Parameter Modulation and Control Analysis

We have explicitly introduced dimensionless modulation parameters $\alpha_{v_{j}}, \alpha_{D_{i}}$, and $\alpha_{f_{i}}$, through which the system can be modified. For the formulation and interpretation of control theorems associated with diffusion we need to introduce two additional forms of modulation, associated with space and time, in the following way. We assume that the spatial and temporal variables $x_{1}, x_{2}, x_{3}$, and $t$, are related to a reference spatial and temporal frame $\xi_{1}, \xi_{2}, \xi_{3}$, and $\tau$, via dimensionless modulation parameters $\alpha_{L}$ and $\alpha_{t}$ :

$$
x_{1}=\alpha_{L} \xi_{1}, \quad x_{2}=\alpha_{L} \xi_{2}, \quad x_{3}=\alpha_{L} \xi_{3}, \quad \text { and } \quad t=\alpha_{t} \tau .
$$

Note that a reference time interval $\left[0, \tau_{\text {end }}\right]$ is rescaled to an actual time interval $\left[0, \alpha_{t} \tau_{\text {end }}\right]=\alpha_{t}\left[0, \tau_{\text {end }}\right]$; and similarly, the reference volume $V$ is scaled to an actual volume $\alpha_{L} V$ (Figure 1 ).

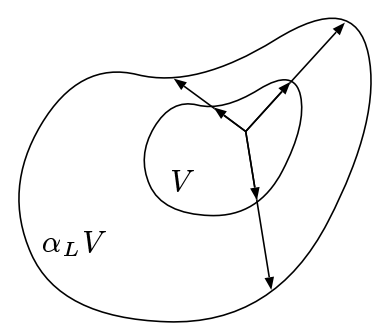

Figure 1: The domain $V$ is scaled by geometric multiplication

We shall be explicit about the cause-and-effect relationship that exists between parameters and state. The parameters are the kinetic constants in rate functions $v_{j}$ and $f_{i}$ and diffusion coefficients $D_{i}$, together with their modulators $\alpha_{v_{j}}, \alpha_{D_{i}}$, and $\alpha_{f_{i}}$. We also consider the volume $V$ and the time interval $\left[0, \tau_{\text {end }}\right]$ as parameters, together with the space and time modulators $\alpha_{L}$ and $\alpha_{t}$. A final 'parameter' that we need to take into account is the distribution of all species in space at the initial time.

For a given choice of all parameters the concentration of each of the species $Y_{i}$ is determined uniquely as a function of space and time, according to Eqs. 2.2 and 2.4. The choice of parameters yields a a unique set of functions $c_{i}(x, t)$, each defined at every point $x \in \alpha_{L} V$ and at each time $t \in\left[0, \alpha_{t} \tau_{\text {end }}\right]$.

Note that the functions $c_{i}$ are defined on different domains for different $\alpha_{L}$ and $\alpha_{t}$. For comparison purposes it will be more convenient to think of $c_{i}$ as functions of the reference coordinates $\xi$ and $\tau$ and the modulation parameters $\alpha$, i.e. $c_{i}(\xi, \tau, \alpha)$, where $\xi \in V, \tau \in\left[0, \tau_{\text {end }}\right]$, and $\alpha=\left(\alpha_{v_{j}}, \alpha_{D_{i}}, \alpha_{f_{i}}, \alpha_{L}, \alpha_{t}\right)$. The functions $c_{i}(\xi, \tau, \alpha)$ determine the state of the system associated with the choice of parameters.

The change of coordinates, from $(x, t)$ to $(\xi, \tau)$, induces a small modification in Eqs. 2.2 and 2.4, which now become

$$
\begin{aligned}
& \alpha_{t}^{-1} \frac{\partial}{\partial \tau} c_{i}-\alpha_{L}^{-2} \alpha_{D_{i}} D_{i} \Delta c_{i}=\sum_{j=1}^{m} N_{i j} \alpha_{v_{j}} v_{j}, \quad \xi \in V, \quad 0<\tau<\tau_{\text {end }} \\
& -\alpha_{L}^{-1} \alpha_{D_{i}} D_{i} \frac{\partial c_{i}}{\partial \nu}=\alpha_{f_{i}} f_{i} \quad \xi \in S, \quad 0<\tau<\tau_{\text {end }}
\end{aligned}
$$

Note that the differentiation in the operators $\Delta$ and $\partial / \partial \nu$ is now with respect to $\xi$. The definition of the flux, however, remains unchanged under this change of coordinates, because of the surface averaging:

$$
J=\frac{\alpha_{f_{1}}}{|S|} \int_{S} f_{1} d S
$$


In the vein of Metabolic Control Analysis we will consider a reference state associated with the choice $\alpha_{v_{j}}=\alpha_{D_{i}}=\alpha_{f_{i}}=\alpha_{L}=\alpha_{t}=1$ and a second state, resulting from an infinitesimal change in one of these modulators. The difference between the two states will be taken to characterize the control that the modulated parameter has on the state of the system. The dimensionless parameter $\alpha_{v_{j}}$ is the tool we shall use to modulate the activity of reaction $v_{j}$. Departures of $\alpha_{v_{j}}$ from 1 correspond to a modulation of enzyme catalytic activity or concentration, or to a proportional modulation of all rate constants of the reaction step if it is not enzyme catalyzed (this modulation leaves the equilibrium constant unchanged, for details see (Kholodenko and Westerhoff 1993, 1994, 1995), where this approach was introduced). For instance, if reaction 2.1 is a protein-protein interaction (a central reaction in signaling networks), the rate $v_{j}$ depends on two kinetic constants, $k_{\text {on }}$ and $k_{\text {off }}$, and a change in $\alpha_{v_{j}}$ corresponds to equal proportional change of both constants that leaves the dissociation (equilibrium) constant $K_{d}$ unchanged. The ratio of the fractional change in a state variable of the reaction network, such as $J$ or $c_{k}$, and the fractional change in $\alpha_{v_{j}}$ determines the control coefficient with respect to reaction rate $v_{j}$ (in the limit of infinitesimally small changes):

$$
C_{v_{j}}^{J}=\left.\frac{d \ln J}{d \ln \alpha_{v_{j}}}\right|_{\alpha_{v_{j}}=1}, \quad C_{v_{j}}^{c_{k}}=\left.\frac{d \ln c_{k}}{d \ln \alpha_{v_{j}}}\right|_{\alpha_{v_{j}}=1},
$$

where $\alpha_{v_{j}}=1$ corresponds to the reference state (all other parameters are assumed fixed). Similarly, considering two states corresponding to small modulations in dimensionless parameters, $\alpha_{f_{i}}$ and $\alpha_{D_{i}}$, we define the control coefficients with respect to transport reaction and diffusion, $C_{f_{i}}^{J}$ and $C_{D_{i}}^{J}$.

An intrinsically novel control coefficient emerges as the heterogeneous spatial organization of cellular reaction network is taken into account. This coefficient is obtained when the size $(L)$ of the system is modified through a modulation of the dimensionless parameter $\alpha_{L}$, as shown schematically in Fig. 1, whilst keeping fixed all other parameters. In a cell setting, these parameters include the volume and surface concentrations of enzymes and other molecular forms, the total concentrations of which are conserved (for instance, the total amount of phosphorylated and unphosphorylated forms of a protein) in network reactions described by Eqs. 2.7 and 2.8. Therefore, increasing the cell size also implies adding conserved chemical moieties and additional enzymes both to the bulk aqueous phase and cell membranes. Accordingly, the 'spatial' control coefficient is defined as $C_{L}^{J}=d \ln J / d \ln \alpha_{L}$, where only the spatial variables are modulated, as described above (Eq. 2.6). It is of note, that the parameter $L$ can be interpreted as the characteristic cell size, e.g., the volume to surface ratio, whose fractional changes are equal to the fractional changes in $\alpha_{L}$.

A special case of Eq. 2.7 arises when we only consider steady-state, non-equilibrium configurations; these correspond to solutions $c_{1}(\xi), \ldots, c_{n}(\xi)$ of the steady-state equations

$$
-\alpha_{L}^{-2} \alpha_{D_{i}} D_{i} \Delta c_{i}=\sum_{j=1}^{m} N_{i j} \alpha_{v_{j}} v_{j}, \quad \xi \in V,
$$

with boundary conditions 2.8. For the purposes of this paper we assume that solutions of this set of equations are locally unique and asymptotically stable.

\section{RESULTS: THE FIRST SUMMATION THEOREM}

In a metabolic network where concentrations are homogeneous, the sum of the flux control by all biochemical reactions equals 1 (Kacser and Burns 1973; Heinrich and Rapoport 1973). We generalize this summation theorem for systems where concentrations may not be homogeneous and where transport occurs - here the sum over all the reactive processes need not be 1 . The flux may be partially controlled by diffusion or by transport and it is because of this control that the classical summation theorem no longer holds. The generalized summation theorem takes the form

$$
\sum_{i=1}^{n} C_{D_{i}}^{J}+\sum_{j=1}^{m} C_{v_{j}}^{J}+\sum_{i=1}^{n} C_{f_{i}}^{J}=1
$$


In words this theorem reads: the total control by all diffusion, reaction, and transport processes on any steady state flux equals 1. The proof of Eq. 3.1 is based on Euler's theorem on homogeneous function, which can be stated as follows. Let $g$ be a function of $p_{1}, \ldots, p_{n}$, such that for all $p_{i}$ and for all $\lambda>0$,

$$
g\left(\lambda^{\beta_{1}} p_{1}, \ldots, \lambda^{\beta_{n}} p_{n}\right)=\lambda^{\gamma} g\left(p_{1}, \ldots, p_{n}\right)
$$

for some $\beta_{1}, \ldots, \beta_{n}, \gamma \in \mathbb{R}$, i.e., $g\left(p_{1}, \ldots, p_{n}\right)$ is the same function after the transformation

$$
\tilde{g}=\lambda^{\gamma} g, \quad \tilde{p}_{i}=\lambda^{\beta_{i}} p_{i} .
$$

Then

$$
\sum_{i=1}^{n} \beta_{i} \frac{\partial \ln |g|}{\partial \ln p_{i}}=\gamma
$$

It may be noted that for some of the parameters $p_{i}, \beta_{i}$ may equal zero. These parameters are not modulated in Eq. 3.3, and Eq. 3.4 shows that these parameters are absent from the summation theorem.

For $\gamma$ and all $\beta_{i}$ equal to 1, Eq. 3.4 reminds of the flux control summation theorem of Metabolic Control Analysis (Kacser and Burns 1973; Heinrich and Rapoport 1973), but it may not be clear $a$ priori what function and which parameters should be considered. When searching for other than the traditional summation theorems, a strategy may prove useful. One strategy is that of leaving the system in essence in the same state (cf. (Kacser and Burns 1973)). In terms of the system under consideration this translates into the concentrations $c_{i}(\xi, \tau)$ remaining the same when the parameters $p_{i}$ of Eq. 3.3 are changed.

In order to implement the above strategy and examine under what type of parameter changes $c_{i}(\xi, \tau)$ might be constant, one may inspect the equations that define $c_{i}$. For steady states Eqs. 2.10 and 2.8 define $c_{i}(\xi)$. Multiplying both equations by the same factor $\lambda$ should leave their solutions unchanged. Consequently, multiplying all $\alpha_{D_{i}}$, all $\alpha_{v_{j}}$, and all $\alpha_{f_{i}}$ by that same factor should not change the solutions $c_{i}(\xi)$ either: the terms in the equations are homogeneous functions of first order of the parameters $\alpha_{D_{i}}, \alpha_{v_{j}}$, and $\alpha_{f_{i}}$. When only these parameters are modulated, the specific transport rate remains unaltered, because the metabolite concentrations remain constant and neither $\alpha_{D_{i}}, \alpha_{v_{j}}$, or $\alpha_{f_{i}}$ occurs in the function $f_{i}\left(c_{1}, \ldots, c_{n}\right)$. Consequently $f_{i}$ remains unchanged, and therefore we can calculate the corresponding flux as a function of $\lambda$,

$$
J(\lambda)=\lambda \frac{\alpha_{f_{1}}}{|S|} \int_{S} f_{1}=\lambda J(1) .
$$

We can reformulate this result as follows: we have shown that the flux $J$, as a function of the parameters $\alpha_{D_{i}}, \alpha_{v_{j}}$, and $\alpha_{f_{i}}$, satisfies

$$
\begin{aligned}
J\left(\lambda \alpha_{D_{1}}, \ldots, \lambda \alpha_{D_{n}}, \lambda \alpha_{v_{1}}, \ldots, \lambda \alpha_{v_{m}}, \lambda \alpha_{f_{1}}, \ldots, \lambda \alpha_{f_{n}}\right) & \\
& =\lambda J\left(\alpha_{D_{1}}, \ldots, \alpha_{D_{n}}, \alpha_{v_{1}}, \ldots, \alpha_{v_{m}}, \alpha_{f_{1}}, \ldots, \alpha_{f_{n}}\right) .
\end{aligned}
$$

Equality 3.1 now follows from Eq. 3.4.

It should be remembered that the parameters $\alpha_{D_{i}}, \alpha_{v_{j}}$, and $\alpha_{f_{i}}$ represent the activities of diffusion, chemical, and transport processes, respectively. Consequently, Eq. 3.1 really refers to a theorem concerning the control by diffusion, chemistry and transport. It is an extension, both in concept and in proof, of the familiar flux control summation theorem of Metabolic Control Analysis. The latter only dealt with the middle terms of the left-hand side and then only the enzyme catalyzed reactions thereof, it assumed concentrations to be homogeneous in space, and neglected explicit transport terms. 
A further extension to the above methodology will generalize the above summation theorem to systems that depend on time: Dropping the assumption of steady state, Eqs. 2.7 and 2.8 become the equations that define $c_{i}(\xi, \tau)$. Multiplying both these equations by the same factor $\lambda$ again leaves their solutions unchanged. Consequently:

$$
-C_{t}^{J(\tau)}+\sum_{i=1}^{n} C_{D_{i}}^{J(\tau)}+\sum_{j=1}^{m} C_{v_{j}}^{J(\tau)}+\sum_{i=1}^{n} C_{f_{i}}^{J(\tau)}=1 .
$$

Here $C_{t}^{J(\tau)}$ coincides with the time-control coefficient defined in (Acerenza et al. 1989). How can we understand this theorem and in particular this new time-control coefficient? Let us first consider the situation that would ensue from simultaneously and equally increasing all the parameters $\alpha_{D_{i}}, \alpha_{v_{j}}$, and $\alpha_{f_{i}}$ by $1 \%$ in the absence of an increase in $\alpha_{t}$. Because all activities increase, also the flux $J$ should increase. However, the system should also change more quickly in time, i.e. the increase in magnitude of $J$ should occur earlier. To obtain a proportionate increase in $J$, one should look at an earlier point in time, i.e. time should be $1 \%$ earlier, or, keeping in mind that $t=\alpha_{t} T, \alpha_{t}=0.99$.

$C_{t}^{J(\tau)}$ should not be confused with the time-dependent control coefficients defined by (Westerhoff and van Dam 1987; Heinrich and Reder 1991). For a further discussion of the distinctions, see also (Kholodenko et al. 1997) for the control analysis of relaxations in the vicinity of the steady state.

Often, as time goes to infinity, the system relaxes to a steady state. The control by time on the flux then reduces to zero and the summation theorem for the time dependent control coefficients reduces to the one for steady state control coefficients (Eq. 3.1).

The summation theorem 3.5 is more general than suggested by the above derivation. It can be derived without requiring explicit equations for the time evolution of the system, such as Eqs. 2.7 and 2.8. The proof then considers an actual physical transformation of the system under consideration: all the time dependent elemental processes are increased by the same factor $\lambda$. This implies that everything happens in the same way, but faster by that same factor $\lambda$. We represent this concept of 'faster' by the parameter $\alpha_{t}$, as above (since $t=\alpha_{t} \tau, \alpha_{t}<1$ corresponds to 'faster', and $\alpha_{t}>1$ to 'slower'). Writing the parameter-dependence explicitly,

$$
c_{i}(\xi, \tau)=c_{i}\left(\xi, \tau ; \alpha_{D_{i}}, \alpha_{v_{j}}, \alpha_{f_{i}}, \alpha_{L}, \alpha_{t}\right)
$$

we have

$$
c_{i}\left(\xi, \tau ; \lambda \alpha_{D_{i}}, \lambda \alpha_{v_{j}}, \lambda \alpha_{f_{i}}, \alpha_{L}, \frac{\alpha_{t}}{\lambda}\right)=c_{i}\left(\xi, \tau ; \alpha_{D_{i}}, \alpha_{v_{j}}, \alpha_{f_{i}}, \alpha_{f_{i}}, \alpha_{t}\right)
$$

or in other words, $c_{i}$ is homogeneous of zeroth order in $\alpha_{D_{i}}, \alpha_{v_{j}}, \alpha_{f_{i}}$, and $1 / \alpha_{t}$. Using Eq. 3.4 this yields the summation theorem for concentrations,

$$
-C_{t}^{c_{k}}+\sum_{i=1}^{n} C_{D_{i}}^{c_{k}}+\sum_{j=1}^{m} C_{v_{j}}^{c_{k}}+\sum_{i=1}^{n} C_{f_{i}}^{c_{k}}=0 .
$$

where $c_{k}$ is short for $c_{k}(\xi, \tau)$. A similar result for the flux,

$$
J\left(\tau ; \lambda \alpha_{D_{i}}, \lambda \alpha_{v_{j}}, \lambda \alpha_{f_{i}}, \frac{\alpha_{t}}{\lambda}\right)=\lambda J\left(\tau ; \alpha_{D_{i}}, \alpha_{v_{j}}, \alpha_{f_{i}}, \alpha_{t}\right),
$$

shows that the flux is homogeneous of first order in $\alpha_{D_{i}}, \alpha_{v_{j}}, \alpha_{f_{i}}$, and $1 / \alpha_{t}$, resulting in the summation theorem Eq. 3.5.

\section{A NOVEL SUMmation THEOREM}

The above theorem is an extension of the theorem of (Acerenza et al. 1989) to cases with transport and diffusion. The theorem we shall develop now is intrinsically new as its origin lies in spatial aspects 
that have hitherto been neglected in Metabolic Control Analysis (Fell 1997). The strategy we follow is again to consider a transformation for which the system properties should not change. We first discuss the nature of diffusion, as illustrated by Einstein's diffusion equation:

$$
\delta x=\sqrt{n_{D} D \delta t}
$$

where $n_{D}$ is a number of order 1 that depends on the dimensionality. In words, the displacement due to diffusion increases with the square root of the diffusion coefficient. Consequently, increasing all diffusion coefficients by $\lambda^{2}$ should increase all displacement by the factor $\lambda$.

This becomes obvious when considering Eq. 2.7. Replacing $\alpha_{D_{i}}$ by $\lambda^{2} \alpha_{D_{i}}$ and $\alpha_{L}$ by $\lambda \alpha_{L}$ leaves Eq. 2.7 unchanged. The boundary condition 2.8 in a natural way suggests replacing $\alpha_{f_{i}}$ by $\lambda \alpha_{f_{i}}$, so that this equation is also essentially unchanged. As a result this choice of parameters leaves the solution $c_{i}$ invariant:

$$
c_{i}\left(\xi, \tau ; \lambda^{2} \alpha_{D_{i}}, \alpha_{v_{j}}, \lambda \alpha_{f_{i}}, \lambda \alpha_{L}, \alpha_{t}\right)=c_{i}\left(\xi, \tau ; \alpha_{D_{i}}, \alpha_{v_{j}}, \alpha_{f_{i}}, \alpha_{L}, \alpha_{t}\right) .
$$

We immediately deduce the second summation theorem for concentrations,

$$
2 \sum_{i=1}^{n} C_{D_{i}}^{c}+\sum_{i=1}^{n} C_{f_{i}}^{c}+C_{L}^{c}=0
$$

where $c$ stands for any $c_{i}(\xi, \tau)$. Similarly, since the flux satisfies

$$
J\left(\tau ; \lambda^{2} \alpha_{D_{i}}, \alpha_{v_{j}}, \lambda \alpha_{f_{i}}, \lambda \alpha_{L}, \alpha_{t}\right)=\lambda J\left(\tau ; \alpha_{D_{i}}, \alpha_{v_{j}}, \alpha_{f_{i}}, \alpha_{L}, \alpha_{t}\right),
$$

the associated summation theorem results

$$
2 \sum_{i=1}^{n} C_{D_{i}}^{J(\tau)}+\sum_{i=1}^{n} C_{f_{i}}^{J(\tau)}+C_{L}^{J(\tau)}=1 .
$$

Naturally, if we only consider steady states, then $c_{i}(\xi, \tau)$ reduces to $c_{i}(\xi)$, and $J(\tau)$ to $J$.

The modulation of $V$, via $\alpha_{L}$, implicated in the evaluation of the corresponding control coefficient is one in which the size of the system changes; it is important, however, that the system size changes whilst keeping the volume concentration of enzymes in the bulk aqueous phase constant and the surface concentrations of the membrane enzymes constant.

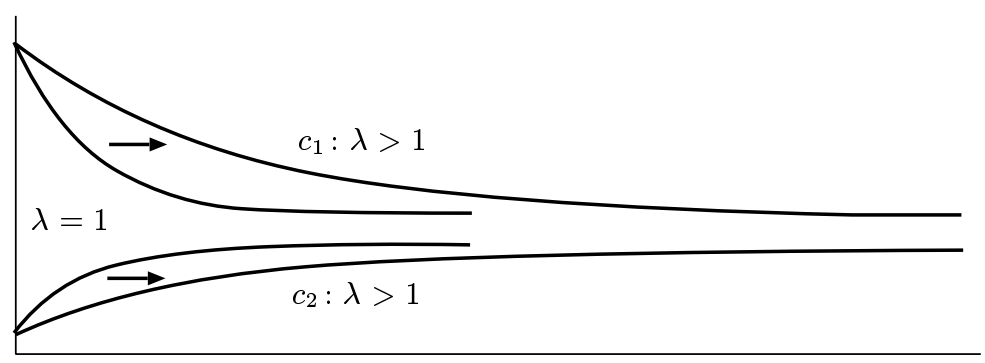

Figure 2: The scaling in $\lambda$ for a two-species system

To illustrate the modulation introduced above, consider the case of a one-dimensional spatial domain of the form $(0, \infty)$; the membrane is thought to be at $x=0$, and the cell is deemed so large that the 'other end' is effectively at infinity. For such a 'half-infinite' cell, in non-equilibrium steady-state, Eq. 4.3 reduces to

$$
2 \sum_{i=1}^{n} C_{D_{i}}^{J}+\sum_{i=1}^{n} C_{f_{i}}^{J}=1
$$


where $J$ is the flux at the border $(x=0) ; C_{L}^{J}$ vanishes as a result of the infinite size. Figure 2 shows how the solution $c_{i}(x)$ depends on the parameter modulation by the factor $\lambda$ given in Eq. 4.1. Note that here $c_{i}$ is plotted as a function of $x$; when plotted as a function of $\xi$, the graphs for different $\lambda$ coincide (cf. 4.1).

\section{An EXPLICIT EXAMPLE SYSTEM}

For certain simple cases control coefficients can be calculated explicitly. We do so here for a kinase/phosphatase example that we also discussed in (Brown and Kholodenko 1999; Kholodenko et al. 2000a). There are two species, $Y$ and $Y P$, which are the unphosphorylated and phosphorylated form of a given protein; the kinase converts $Y$ into $Y P$, and the phosphatase does the reverse.

Besides providing an illustration of the concepts and results discussed above we wish to demonstrate the influence of the system geometry on the control coefficients. We consider two cases:

1. $V$ is bounded by two parallel membranes; the kinase reaction is localized to one membrane, the phosphatase to the other (Figure 3);

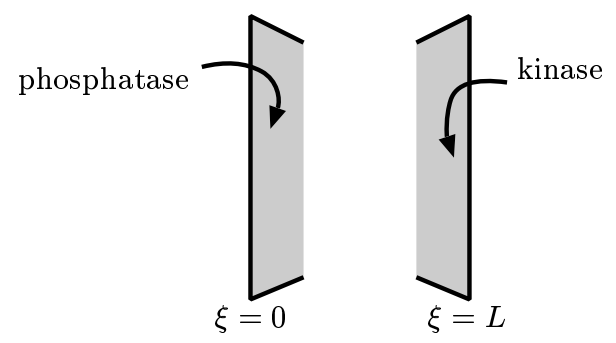

Figure 3: Geometry 1: a slice bounded by parallel membranes

1. $V$ is a spherical region (e.g. a cell or an organelle) bounded by a membrane; the kinase is localized at the membrane, but the phosphatase is distributed throughout the bulk (Figure 4).

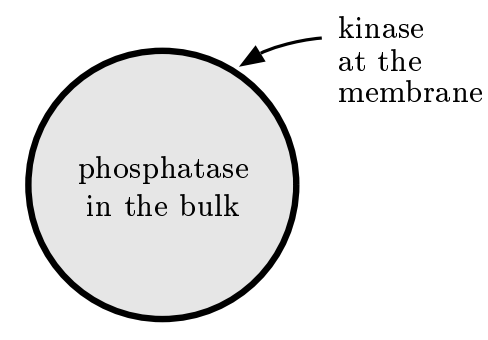

Figure 4: Geometry 2: a spherical cell

As we shall see below, in the case of parallel membranes the size control coefficient $C_{L}^{J}$ is negative: an increase in the distance between the membranes reduces the flux through the system. However, when the phosphatase is not membrane-bound but distributed, as in the second case, increasing the system size actually increases the flux: $C_{L}^{J}$ is positive.

\subsection{Two parallel membranes}

We assume that the system is large with respect to the distance $L$ between the membranes and therefore adopt a one-dimensional formulation. We also restrict the study to (non-equilibrium) steady state. 
Since no reaction takes place in the bulk, the equations satisfied by $Y$ and $Y P$ in the bulk (Eq. 2.10) reduce to

$$
-\alpha_{L}^{-2} \alpha_{D} D Y P^{\prime \prime}=-\alpha_{L}^{-2} \alpha_{D} D Y^{\prime \prime}=0, \quad 0<\xi<L .
$$

Note that the spatial variable is $\xi$, as discussed above; the physical variable $x$ is given by $x=\alpha_{L} \xi$.

For the kinase and phosphatase reactions we assume the rate functions

$$
\alpha_{k} k_{k}\left(\kappa_{k} Y-Y P\right) \quad \text { and } \quad \alpha_{p} k_{p}\left(Y P-\kappa_{p} Y\right) \text {. }
$$

Note the dimensionality of $k_{k, p}$ : the rate functions are surface fluxes, but $Y P$ and $Y$ are bulk concentrations; $k_{k}$ and $k_{p}$ therefore have dimension length/time. The coefficients $\alpha_{k, p}$ and $\kappa_{k, p}$ are dimensionless.

The kinase and phosphatase reactions both enter the description as boundary conditions (cf. 2.8):

$$
\begin{aligned}
\alpha_{L}^{-1} \alpha_{D} D Y P^{\prime} & =\alpha_{p} k_{p}\left(Y P-\kappa_{p} Y\right) \\
\alpha_{L}^{-1} \alpha_{D} D Y^{\prime} & =-\alpha_{p} k_{p}\left(Y P-\kappa_{p} Y\right) \\
-\alpha_{L}^{-1} \alpha_{D} D Y P^{\prime} & =-\alpha_{k} k_{k}\left(\kappa_{k} Y-Y P\right) \\
-\alpha_{L}^{-1} \alpha_{D} D Y^{\prime} & =\alpha_{k} k_{k}\left(\kappa_{k} Y-Y P\right)
\end{aligned} \quad \text { at } \xi=0 \text { (phosphatase) }
$$

Equation 5.1 implies that the spatial gradients of $Y P$ and $Y$ (i.e. $Y P^{\prime}$ and $Y^{\prime}$ ) are constant in $\xi$; let us set $\delta=Y P^{\prime}$. From the solution of equations (5.1-5.3) the relevant information for our purposes is $\delta$ :

$$
\delta=\frac{M\left(\beta_{p} \alpha_{k} \kappa_{k}-\beta_{k} \alpha_{p} \kappa_{p}\right)}{\alpha_{L}^{-1} \alpha_{D} D\left(\beta_{k}+\beta_{p}\right)+L \beta_{k} \beta_{p}} .
$$

Here $M$ is the total concentration $Y+Y P$, which is independent of $\xi$ by Eq. 5.1, and

$$
\beta_{k}=\alpha_{k} k_{k}\left(1+\kappa_{k}\right) \quad \text { and } \quad \beta_{p}=\alpha_{p} k_{p}\left(1+\kappa_{p}\right) .
$$

Because of the spatial separation of the reactions the flux as defined by Eq. 2.5 equals the diffusive flux,

$$
J=-\alpha_{L}^{-1} \alpha_{D} D Y P^{\prime}=-\alpha_{L}^{-1} \alpha_{D} D \delta .
$$

From Eqs. 5.4 and 5.5 we can determine the control coefficients of the flux with respect to the kinase and phosphatase reactions and to diffusion, as well as the 'length'-control coefficient: ${ }^{1}$

$$
\begin{aligned}
C_{k}^{J} & =\frac{D \beta_{p}}{D\left(\beta_{k}+\beta_{p}\right)+L \beta_{k} \beta_{p}}, \\
C_{p}^{J} & =\frac{D \beta_{k}}{D\left(\beta_{k}+\beta_{p}\right)+L \beta_{k} \beta_{p}}, \\
C_{D}^{J} & =\frac{L \beta_{k} \beta_{p}}{D\left(\beta_{k}+\beta_{p}\right)+L \beta_{k} \beta_{p}}, \\
C_{L}^{J} & =-\frac{L \beta_{k} \beta_{p}}{D\left(\beta_{k}+\beta_{p}\right)+L \beta_{k} \beta_{p}} .
\end{aligned}
$$

Clearly these expressions satisfy the summation theorems Eqs. 3.1 and 4.3:

$$
\begin{aligned}
& C_{D}^{J}+C_{k}^{J}+C_{p}^{J}=1 \\
& 2 C_{D}^{J}+C_{k}^{J}+C_{p}^{J}+C_{L}^{J}=1
\end{aligned}
$$

\footnotetext{
${ }^{1}$ Note that these formulas contain no $\alpha$ 's, since they result from evaluation at $\alpha_{L}=\alpha_{D}=\alpha_{k}=\alpha_{p}=1$; consequently, $\beta_{k, p}=k_{k, p}\left(1+\kappa_{k, p}\right)$.
} 
Note that both the kinase and the phosphatase reaction are boundary effects, and therefore give rise to boundary control coefficients $C_{f_{i}}^{J}$ of Eqs. 3.1 and 4.3 .

For concentration control we choose to consider not a pointwise concentration, but the difference between concentrations at opposite ends:

$$
Y P(L)-Y P(0)=L \delta
$$

Denoting the control coefficients with respect to this quantity as $C^{c}$, we have

$$
\begin{aligned}
C_{k}^{c} & =\frac{D \beta_{p}}{D\left(\beta_{k}+\beta_{p}\right)+L \beta_{k} \beta_{p}}, \\
C_{p}^{c} & =\frac{D \beta_{k}}{D\left(\beta_{k}+\beta_{p}\right)+L \beta_{k} \beta_{p}}, \\
C_{D}^{c} & =-\frac{D\left(\beta_{k}+\beta_{p}\right)}{D\left(\beta_{k}+\beta_{p}\right)+L \beta_{k} \beta_{p}}, \\
C_{L}^{c} & =\frac{D\left(\beta_{k}+\beta_{p}\right)}{D\left(\beta_{k}+\beta_{p}\right)+L \beta_{k} \beta_{p}} .
\end{aligned}
$$

An example of the values of these control coefficients is given in Figure 5.
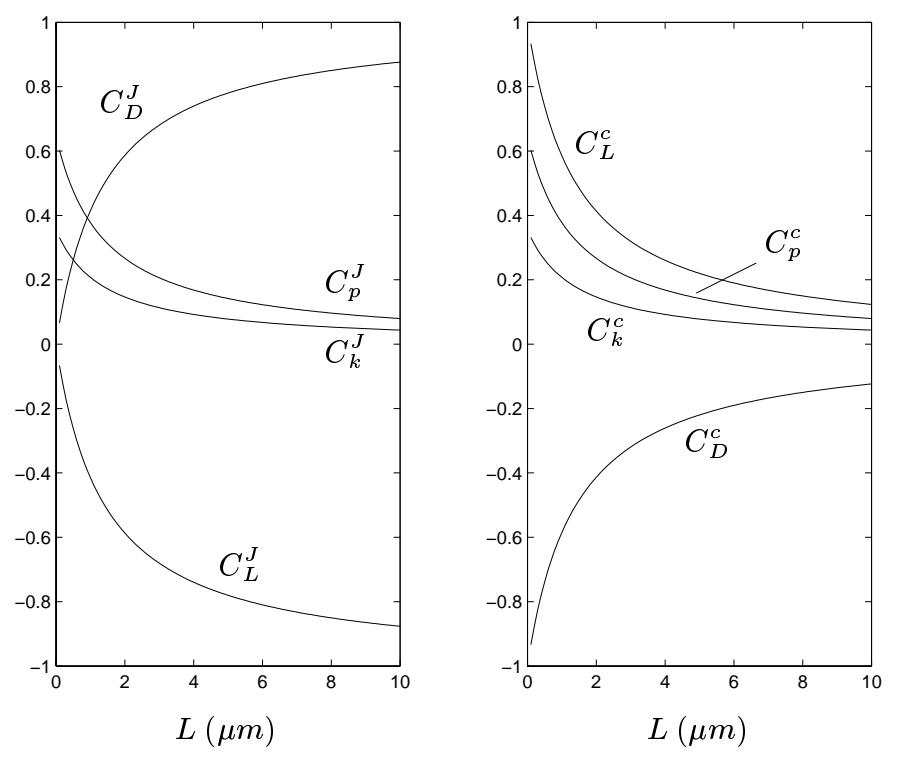

Figure 5: Values of control coefficients as a function of $L$ for the first example (parallel membranes at distance $L$, the kinase and phosphatase are localized at different membranes; $D=1 \mu m^{2} / s, k_{p}=$ $\left.k_{k}=1 / s, \kappa_{p}=0.1, \kappa_{k}=10\right)$.

\subsection{A spherical cell}

For the second example we consider the same system as above, but now in a spherical cell of radius $L$. We assume spherical symmetry throughout.

The kinase reaction is again localized at the membrane, and therefore results in a boundary condition

$$
\begin{aligned}
-\alpha_{L}^{-1} \alpha_{D} D Y P^{\prime} & =\alpha_{k} k_{k}\left(Y P-\kappa_{k} Y\right), \\
-\alpha_{L}^{-1} \alpha_{D} D Y^{\prime} & =-\alpha_{k} k_{k}\left(Y P-\kappa_{k} Y\right), \quad \text { at } \xi=L \text { (kinase). }
\end{aligned}
$$


The spatial variable is again $\xi$, but it now represents a radial coordinate. The phosphatase reaction, which in this example is distributed throughout the bulk, now enters as a right-hand side in the differential equation for $Y$ and $Y P$ (cf. 2.10):

$$
\begin{aligned}
& -\alpha_{L}^{-2} \alpha_{D} D \xi^{-2}\left(\xi^{2} Y P^{\prime}\right)^{\prime}=-\alpha_{p} k_{p}\left(Y P-\kappa_{p} Y\right), \\
& -\alpha_{L}^{-2} \alpha_{D} D \xi^{-2}\left(\xi^{2} Y^{\prime}\right)^{\prime}=\alpha_{p} k_{p}\left(Y P-\kappa_{p} Y\right),
\end{aligned}
$$

Again note the dimensions of $k_{k}$ and $k_{p}$ : similar to the previous example, $k_{k}$ has dimension length/time; but here $k_{p}$ converts a bulk concentration into a bulk flux, and therefore $k_{p}$ has the (more familiar) dimension $1 /$ time. As before, the total concentration is independent of $\xi$, and denoted by $M$ :

$$
M=Y P+Y
$$

As a first step we investigate different boundary conditions, where we prescribe the flux $J$ :

$$
-\alpha_{L}^{-1} \alpha_{D} D Y P^{\prime}=J=\alpha_{L}^{-1} \alpha_{D} D Y^{\prime},
$$

at $\xi=L$. For this choice of boundary conditions we can solve the system exactly. Set $u=Y P-\kappa_{p} Y$; then $u$ satisfies the equation

$$
-\alpha_{L}^{-2} \alpha_{D} D \xi^{-2}\left(\xi^{2} u^{\prime}\right)^{\prime}=-\alpha_{p} k_{p}\left(1+\kappa_{p}\right) u,
$$

with boundary condition

$$
-\alpha_{L}^{-1} \alpha_{D} D u^{\prime}(L)=\left(1+\kappa_{p}\right) J .
$$

We find that $u$ is given by

$$
u(\xi)=-J \frac{1+\kappa_{p}}{\alpha_{L}^{-1} \alpha_{D} D} \frac{L^{2}}{\gamma L \cosh \gamma L-\sinh \gamma L} \frac{\sinh \gamma \xi}{\xi},
$$

where the inverse length scale $\gamma$ is defined by

$$
\gamma^{2}=\alpha_{L}^{2} \frac{\alpha_{p} k_{p}\left(1+\kappa_{p}\right)}{\alpha_{D} D}
$$

It follows that

$$
\begin{aligned}
Y P(\xi) & =\frac{\kappa_{p}}{1+\kappa_{p}} M+\frac{1}{1+\kappa_{p}} u(\xi) \\
Y(\xi) & =\frac{1}{1+\kappa_{p}} M-\frac{1}{1+\kappa_{p}} u(\xi) .
\end{aligned}
$$

To return to the boundary conditions 5.6 we equate the flux $J$ in Eq. 5.8 with the right-hand side of Eq. 5.6:

$$
J=\alpha_{k} k_{k}\left(Y P(L)-\kappa_{k} Y(L)\right)=\alpha_{k} k_{k}\left(\frac{1+\kappa_{k}}{1+\kappa_{p}} u(L)+\frac{\kappa_{p}-\kappa_{k}}{1+\kappa_{p}} M\right)
$$

using the expression 5.9 for $u$ this yields

$$
J=\frac{\nu}{1+\beta}, \quad \nu=\alpha_{k} k_{k} M \frac{\kappa_{p}-\kappa_{k}}{1+\kappa_{p}}, \quad \beta=\alpha_{k} k_{k} \frac{1+\kappa_{k}}{\alpha_{L}^{-1} \alpha_{D} D} \frac{L}{\gamma L \operatorname{coth} \gamma L-1}
$$

$(\operatorname{coth} x$ is the hyperbolic cotangent, $\cosh x / \sinh x)$. 
As in the previous example we thus obtain explicit formulas for the control of the various parameters on the flux:

$$
\begin{aligned}
C_{k}^{J} & =\frac{1}{1+\beta} \\
C_{D}^{J} & =\frac{1}{2} \frac{\beta}{1+\beta} \frac{\gamma L \operatorname{coth} \gamma L-2+\gamma^{2} L^{2}\left(\operatorname{coth}^{2} \gamma L-1\right)}{\gamma L \operatorname{coth} \gamma L-1} \\
C_{p}^{J} & =\frac{1}{2} \frac{\beta}{1+\beta} \frac{\gamma L \operatorname{coth} \gamma L-\gamma^{2} L^{2}\left(\operatorname{coth}^{2} \gamma L-1\right)}{\gamma L \operatorname{coth} \gamma L-1} \\
C_{L}^{J} & =\frac{\beta}{1+\beta} \frac{1-\gamma^{2} L^{2}\left(\operatorname{coth}^{2} \gamma L-1\right)}{\gamma L \operatorname{coth} \gamma L-1}
\end{aligned}
$$

As an example of the concentration control values we choose a point at distance $L / 2$ from the center of the cell. Denoting the control coefficients with respect to this quantity as $C^{c}$, we calculate

$$
\begin{aligned}
C_{k}^{c} & =\mu C_{k}^{J} \\
C_{D}^{c} & =\mu\left(C_{D}^{J}+\frac{1}{2} \phi-1\right) \\
C_{p}^{c} & =\mu\left(C_{p}^{J}-\frac{1}{2} \phi\right) \\
C_{L}^{c} & =\mu\left(C_{L}^{J}-\phi+1\right)
\end{aligned}
$$

Here

$$
\mu=\frac{u(L / 2)}{\kappa_{p} M+u(L / 2)} \quad \text { and } \quad \phi=\frac{\gamma^{2} L^{2}}{\gamma L \operatorname{coth} \gamma L-1}-\frac{\gamma L}{2} \operatorname{coth}\left(\frac{\gamma L}{2}\right) .
$$

An example of the values of these control coefficients is given in Figure 6.

\section{Discussion}

Until fairly recently, biochemistry focused more on the time dependent aspects of processes than on the spatial aspects. Exceptions were the membrane-mediated subcompartmentation of cellular metabolism. The cellular subcompartments were mostly considered to be homogeneous in terms of concentrations and the heterogeneity between them was analyzed in terms of the activity of transport catalysts in the membranes (Westerhoff and van Dam 1987). Inhomogeneity of metabolites and even ions within aqueous subcompartments has often been proposed (Kell 1979) but calculations have shown that at least for central metabolic routes aqueous diffusion should be fast on the time scale of the catalytic turnover of the enzymes also given the proximity of the enzyme molecules due to the small sizes of cells (Westerhoff and van Dam 1987; Westerhoff and Welch 1992). Metabolic Control Analysis was developed from this perspective and proved a useful way to rationalize the study of regulation and control of intermediary metabolism (Fell 1997) and free-energy transduction (Westerhoff and van Dam 1987). More than an analysis procedure, Metabolic Control Analysis also provided biochemists with a set of laws (theorems) that govern control of fluxes and concentrations in metabolic networks. One type of these were the summation theorems, stating that the sum of the control by all the individual enzyme catalyzed reactions on flux and concentration (Kacser and Burns 1973; Heinrich and Rapoport 1973), oscillation amplitude (Kholodenko et al. 1997) or relaxation time constant (Heinrich and Reder 1991) should equal 1, 0, 1, and 1 respectively.

Those were the days where biochemistry focused on metabolism. However, only a minor fraction of the known genomes encodes primary metabolism. Much coding capacity is devoted to regulation, 

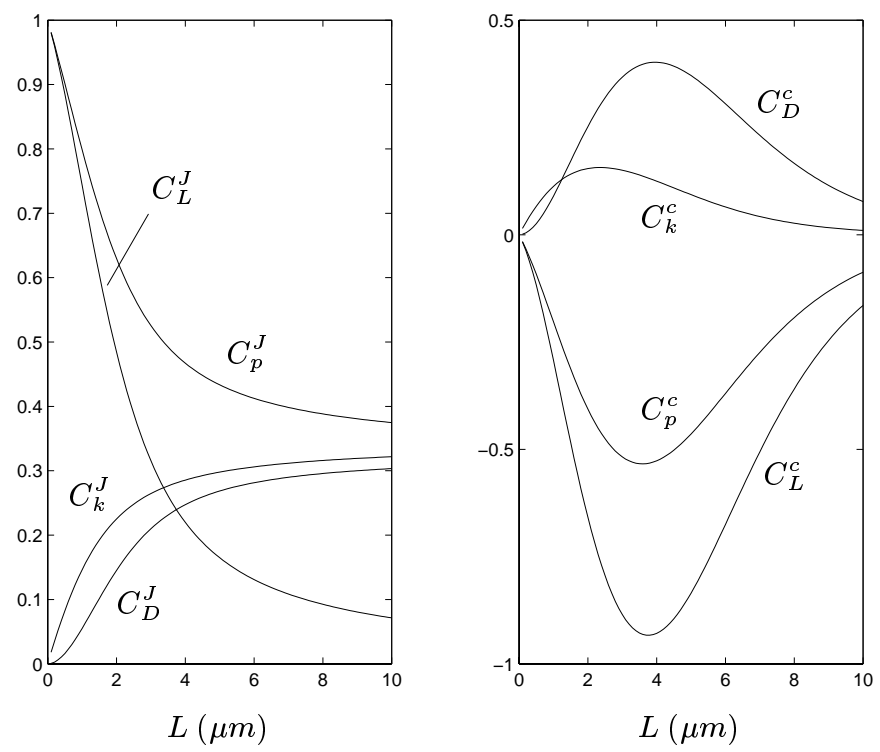

Figure 6: Values of control coefficients as a function of $L$ for the second example (a spherical cell with a kinase on the membrane and a phosphatase in the cytosol; $D=1 \mu \mathrm{m}^{2} / \mathrm{s}, k_{p}=k_{k}=1 / \mathrm{s}, \kappa_{p}=0.1$, $\left.\kappa_{k}=10\right)$. $C^{c}$ denotes control of the concentration measured halfway between the center and the membrane.

in part through signal transduction (Kell 1979; Kholodenko et al. 2000c). Signal transduction has (i) the spatial aspect that many signals arrive at the cell's plasma membrane and have to be transferred to its nuclear DNA, (ii) the added complication that much of the signal is transferred by proteins in direct phosphoryl transfers, rather than with small molecules as messengers, and (iii) the feature that those proteins are often present at a thousand times lower concentrations than the usual metabolite. All these three features work in the direction that might make diffusion of the components limiting in signal transfer. Indeed, it was calculated that for realistic parameter values, concentration gradients in signal (defined as in (Francke et al.)) should arise (Fell 1980; Brown and Kholodenko 1999; Kholodenko et al. 2000a), which might even force the cell to take refuge to alternative mechanisms of movement of the signal proteins (Kholodenko 2002).

With the realization of the possible importance of transport and diffusion for cell biochemistry, there came a need to update Metabolic Control Analysis so as to include the aspects of diffusion. This is what the first part of this paper accomplished for the summation theorem. The result was rather simple, to the sum of the enzyme control coefficients also coefficients for the control by the diffusion of the various species needed to be added, as well as control coefficients for the transport processes and for the non-enzyme-catalyzed chemical reactions, before it amounted to 1 for flux control and 0 for concentration control. This result is perhaps not surprising, but has been overlooked effectively in the literature for quite a while. In studies of the control of growth rate of $E$. coli by the lactose permease, it was found that a sum of 1 could not be found and it was suspected that part of the control resided in diffusion across the outer membrane (Jensen et al. 1993; Dykhuizen et al. 1987). It was found that none of the glycolytic enzymes had significant control on the glycolytic flux in yeast. This was considered a problem until strong indications arose of substantial control in glucose transport into the cells (Reijenga et al. 2001). Thus, if anything, our extension of the summation theorem to include the transport steps may open eyes to the possibility that transport controls metabolic fluxes. Similarly we should expect renewed attention to arise from the other term in our summation theorem, i.e. the one that refers to control by the diffusion. Of course the novelty lies not so much in the possibility 
of such control, but in the fact that it may account for a shortfall of metabolic control from the total of 1 .

We have also formulated the summation theorem for the case of inhomogeneity in both time and space, i.e. where a spatial gradient may be developing over time. In that case diffusion and time turn out to share in the total flux control of 1 . In the case of homogeneity in time (i.e. at steady state), the former contribution disappears, in case of spatial homogeneity the latter. The experimental system that springs to mind is that of Belousov-Zhabotinsky reactions exhibiting time varying spirals (Zhabotinsky and Zaikin 1973), but closer to biology the wave type oscillations in yeast extracts (Mair et al. 2001). The most relevant application may be in developmental biology, but for this an extension to Hierarchical Control Analysis (Snoep et al. 2002) will be needed.

While the first flux summation result, Eq. 3.1, and the first concentration summation result, Eq. 3.6, are a simple extension of existing theory, the summation theorems concerning changes of spatial scale (Eqs. 4.2, 4.3, and 4.4) are fundamentally new, and introduce a concept that is new to Metabolic Control Analysis: control by size.

'Control by the size', the quantity measured by $C_{L}$, is a concept with some interesting properties. For instance, $C_{L}^{J}$ and $C_{L}^{c}$ can be both positive and negative. This is related to the fact that the size of a system can influence the 'productive' capacity of the system in different ways at the same time. Let us examine this for flux control:

- Size can be an obstacle: if material has to travel over a distance $L$, as in the example of parallel membranes (Case 1 of Section 5), then an increase in $L$ implies a decrease of the (average) concentration gradient, and therefore causes a reduction in diffusive flux. This is the dominant effect in the large- $L$ limit of Case 1 , as shown in Fig. 5 , where it leads to a limit value of $C_{L}^{J}$ of -1 .

- On the other hand, size can also be a resource: if a reaction takes place in a bulk region of linear size $L$, then $L$ is also a measure of reactive capacity: an increase in size will result in an increase of total reactive flux. This is demonstrated by the second example, where the input- and output boundaries are co-located and no material is forced to travel over a distance $L$. Here the control by size is positive, and in the limit of small $L$ the control even approaches 1 .

The new summation theorems have a number of interesting implications. Perhaps the most striking one is that they impose an upper limit to the control that diffusion may have on the flux through the system. The first summation theorem suggested that this number be 1, i.e. if the biochemical and the transport processes were in excess and the system were large; the second, however, shows that it is only $1 / 2$ whenever the control by size is positive (i.e. when increasing the size results in a higer flux). Clearly, in such cases there must always be another process controlling flux, in addition to diffusion. In the example of Fig. 6 the other processes were the biochemical reactions.

The example of Figure 2 also shows how changing the diffusion rate alters the concentration profiles. By increasing the diffusion rate we increase the width of a 'boundary layer', in which a significant part of the reactions takes place. As a result the flux is also increased. Note, however, that the relative increase in size of the boundary layer (which scales as $\lambda$, since $x=\alpha_{L} \xi=\lambda \xi$ ) is the square root of the increase of the diffusion coefficients $\left(\alpha_{D_{i}}=\lambda^{2}\right)$. This can be explained intuitively as follows. The size of the boundary layer is determined by counteracting forces; a thin boundary layer provides for fast spatial transport (on the scale of the layer thickness), but little reactive capacity, while for a thick layer the situation is inversed. An increase in diffusion rate is therefore used partially to increase transport rate, and partially to increase reaction capacity. The number $1 / 2$ is the result of this trade-off.

\section{ACKNOWLEDGEMENT}

This work was supported by the RTN network 'Nonlinear Partial Differential Equations describing Front Propagation and other Singular Phenomena', HPRN-CT-2002-00274, and by the National Institute of Health through Grant GM59570. 


\section{REFERENCES}

Acerenza, L., Sauro, H. M., and Kacser, H. 1989. Control analysis of time-dependent metabolic systems. J. Theoret. Biol. 137:423-444

Brown, G. C. and Kholodenko, B. N. 1999. Spatial gradients of cellular phospho-proteins. FEBS Letters 457:452-454

Dykhuizen, D. E., Dean, A. M., and Hartl, D. L. 1987. Metabolic flux and fitness. Genetics 115:25-31

Fell, D. A. 1980. Theoretical analyses of the functioning of the high- and low- $K_{m}$ cyclic nucleotide phosphodiesterases in the regulation of the concentration of adenosine 3',5'-cyclic monophosphate in animal cells. J. Theor. Biol. 84:361-385

—. 1997. Understanding the Control of Metabolism. Portland Press, London

Francke, C., Postma, P. W., Westerhoff, H. V., Blom, J. G., and Peletier, M. A. Why the phosphotransferase system of Escherichia coli escapes the diffusion limitation of signal transduction, transport and metabolism that confronts mammalian cells

Haugh, J. M. and Lauffenburger, D. A. 1997. Physical modulation of introcellular signalling processes by locational regulation. Biophys. J. 72:2014-2031

Heinrich, R. and Rapoport, T. 1973. Linear theory of enzymatic chains; its application for the analysis of the crossover theorem and of the glycolysis of human erythrocytes. Acta Biol. Med. Germ. 31:479-494

Heinrich, R. and Reder, C. 1991. Metabolic control analysis of relaxation processes. J. Theor. Biol. 151:343-350

Jensen, P. R., Westerhoff, H. V., and Michelsen, O. 1993. The use of lac-type promoters in control analysis. Eur. J. Biochem. 211:181-191

Kacser, H. and Acerenza, L. 1993. A universal method for achieving increases in metabolite production. Eur. J. Biochem. 216:361-367

Kacser, H. and Burns, J. 1973. The control of flux. Symp. Soc. Exp. Biol. 27:65-104

Katchalsky, A. and Curran, P. F. 1965. Nonequilibrium Thermodynamics in Biophysics. Harvard

Kell, D. B. 1979. On the functional proton current pathway of electron transport phosphorylation. An electrodic view. Biochim. Biophys. Acta 549:55-99

Kholodenko, B. N. 2002. MAP kinase signalling and endocytic trafficking: A marriage of convenience? Trends Cel. Biol. 12:173-177

Kholodenko, B. N., Brown, G. C., and Hoek, J. B. 2000a. Diffusion control of protein phosphorylation in signal transduction pathways. Biochem. J. 350:901-907

Kholodenko, B. N., Demin, O. V., and Westerhoff, H. V. 1997. Control analysis of periodic phenomena in biological systems. J. Phys. Chem. B101:2070-2081

Kholodenko, B. N., Hoek, J. B., and Westerhoff, H. V. 2000b. Why cytoplasmic signalling proteins should be recruited to cell membranes. Trends Cell Biol. 10:173-178

Kholodenko, B. N. and Westerhoff, H. V. 1993. Metabolic channelling and control of the flux. FEBS Letters 320:71-74

—. 1994. Control theory of one enzyme. Biochim. Biophys. Acta 1208:294-305 
- 1995. The macroworld versus the microworld of biochemical regulation and control. Trends Biochem. Sci. 20:52-54

Kholodenko, B. N., Westerhoff, H. V., Schwaber, J., and Cascante, M. 2000c. Engineering a living cell to desired metabolite concentrations and fluxes: Pathways with multifunctional enzymes. Metabolic Engineering 2:1-13

Mair, T., Warnke, C., and Muller, S. C. 2001. Spatio-temporal dynamics in glycolysis. Faraday Discuss. 120:249-259

Reijenga, K. A., Snoep, J. L., Diderich, J. A., van Verseveld, H. W., Westerhoff, H. V., and Teusink, B. 2001. Control of glycolytic dynamics by hexose transport in Saccharomyces cerevisiae. Biophys. J.

Snoep, J. L., van der Weijden, C. C., Andersen, H. W., Westerhoff, H. V., and Jensen, P. R. 2002. DNA supercoiling in Escherichia coli is under tight and subtle homeostatic control, involving geneexpression and metabolic regulation of both topoisomerase I and DNA gyrase. Eur. J. Biochem. 269:1662-1669

Westerhoff, H. V. and van Dam, K. 1987. Thermodynamics and Control of Biological Free-energy Transduction. Elsevier, Amsterdam

Westerhoff, H. V. and Welch, G. R. 1992. Enzyme organization and the direction of metabolic flow: Physicochemical considerations. Curr. Top. Cell. Regul. 33:361-390

Zhabotinsky, A. M. and Zaikin, A. N. 1973. Autowave processes in a distributed chemical system. J. Theor. Biol. 40:45-61 